\title{
FREIARBEIT IM DAF-UNTERRICHT IN DER GRUNDSCHULE - MÖGLICHKEITEN UND GRENZEN
}

„Sollten Sie auf irgendeine Weise mit Bildung zu tun haben, bleiben Ihnen drei Optionen: Sie können für Veränderungen innerhalb des Systems eintreten, Sie können für eine Veränderung des Systems eintreten oder Sie können die Initiative von außen hineintragen.“ (Aronica und Robinson 2015: xix)

Die DaF-Lehrenden in slowenischen Regelschulen stehen oft vor der Herausforderung, altersgemischte Schülergruppen sowie Lernende mit unterschiedlichen Vorkenntnissen in Deutsch zu unterrichten - vor allem, was Deutsch als nichtobligatorisches Wahlfach anbetrifft. Auch die Autorinnen des nationalen Lehrplans für die zweite Fremdsprache als nichtobligatorisches Wahlfach von der 4. bis zur 9. Klasse (im weiteren Verlauf wird er als Lehrplan bezeichnet) stimmen dem zu (Pevec Semec et al. 2013).

Im vorliegenden Beitrag wird Freiarbeit im DaF-Unterricht aus theoretischer und vor allem aus praktischer Sicht vorgestellt. Es geht um eine Form des offenen Unterrichts, bei der selbständiges, vorwiegend individuelles Lernen in einer vorbereiteten Umgebung im Vordergrund steht. Andere Handlungsformen des offenen Unterrichts sind sowohl Stationenlernen, Arbeit nach Wochenplan, Projektarbeit, als auch der Werkstattunterricht (vgl. Fisgus, Kraft 2012, Peschel 2016). Viele Fachleute stellen fest, dass es keine einheitliche Definition des offenen Unterrichts gibt und dass es nicht um eine Theorie geht, sondern vielmehr um eine Bewegung (Göndör 2013).

Die Freiarbeit kommt in vielen Reformschulen vor. Die italienische Ärztin und Reformpädagogin Maria Montessori (1870-1952) begründete die sog. Vorbereitete Umgebung für die Freiarbeit, der französische Lehrer und Reformpädagoge Célestine Freinet (1896-1966) dagegen den Werkstattunterricht bzw. die Arbeitsecken. Beide sind die Vorgänger des Konstruktivismus, der die Vorstellungen von Lernenden als leeren Gefäßen, die mit Wissen gefüllt werden müssen, ablehnt, und der die Wissenskonstruktion durch Eigenerfahrung mit der Umgebung befürwortet (Marentič Požarnik 2018, Harrer 2015). 
Da der konstruktivistische Lehransatz den Ausgangspunkt der meisten zeitgenössischen pädagogischen Psychologen darstellt (de Corte 2013), wird im vorliegenden Beitrag die praktische Vorstellung der Freiarbeit im Wahlfach Deutsch in der 4. und 5. Klasse der Grundschule an vielen Stellen mit den Ideen Montessoris und Freinets untermauert.

Der didaktische Ansatz stellt die Möglichkeit einer gelungenen Auseinandersetzung mit der Lage, in der sich sowohl viele Lehrende als auch zahlreiche Lernende bei diesem Wahlfach befinden, dar. Zu Beginn werden die Hauptprinzipien der Freiarbeit im Praxisfeld Deutsch als Fremdsprache genauer erörtert. Innerhalb dieses Themas werden drei konkrete DaF-Freiarbeitsmaterialien präsentiert und kommentiert. Anschließend befasst sich der Beitrag mit den Möglichkeiten und Grenzen der Freiarbeit im Deutschunterricht aus der Sicht des Lehrplans, sowie aus dem Blickwinkel anderer beeinflussender Faktoren.

FREIARBEIT IN DER THEORIE UND PRAXIS

\subsection{Vorbereitete Umgebung für den Deutschunterricht in der 4. und 5. Klasse}

Der Termin vorbereitete Umgebung stammt aus der Montessori-Pädagogik und bedeutet, dass der Erwachsene einen Lernraum herstellt, in dem Bedingungen herrschen, die dem Kind freie Bewegung und freie Wahl der Arbeit ermöglichen. Die Hauptmerkmale der vorbereiteten Umgebung sind Ästhetik, Ordnung bzw. Klarheit und Förderung der Unabhängigkeit des Kindes. Der Erwachsene muss sowohl für die Gestaltung des Raumes sorgen als auch die den Kindern angebotenen Materialien gut kennen. Außerdem müssen die Pädagogen über eine innere Sensibilität verfügen, mit deren Hilfe sie zur Erkenntnis gelangen, zu welchem Zeitpunkt die kindliche Arbeit (nicht) unterbrochen werden sollte (Montessori 2009, $2011^{2}$ ).

Deutsch als Wahlfach im Elementarbereich der slowenischen Schulen erfolgt meistens in Gast-Klassenzimmern, was mit sich bringt, dass die Lernumgebung erst kurz vor dem Unterricht vorbereitet werden kann. Die zuvor erstellten Freiarbeitsmaterialien werden auf den Bänken und in den Regalen, die zur Verfügung stehen, ausgelegt. Auch der Fußboden kann als Arbeitsplatz miteinbezogen werden, deswegen sind Teppiche ebenfalls ein Teil der vorbereiteten Umgebung. Außerdem sind ein PC um zum Beispiel Wörter nachzuschlagen und ein Radio mit Kopfhörern für das Hören von kurzen Texten wichtige Elemente der Freiarbeit im Sprachunterricht. Es ist von großer Bedeutung, dass sich die Lernenden im Klassenzimmer alleine zurechtfinden, deswegen ist eine klare

1 Slowenische Übersetzung aus dem Englischen. Die Originalausgabe erschien 1936 unter dem Titel „Il Segreto dell' infanzia“.

2 Slowenische Übersetzung aus dem Englischen. Die Originalausgabe erschien 1952 unter dem Titel „La mente del bambino: mente assorbente“. 
Präsentation der Sprachmaterialien und der Arbeitsweise von entscheidender Bedeutung. Es muss betont werden, dass das Kind das Material nach Gebrauch auf den gleichen Platz verräumt, um für den Nächsten bereit zu stehen. Üblicherweise dauert es einige Unterrichtsstunden bis sich die Kinder an diese Arbeitsweise gewöhnt haben. Brügelmann (1997) schreibt, dass wiederkehrende Aufgabentypen, ähnliche Arbeitsformen mit dem Material, zeitliche und örtliche Strukturen der Arbeit den Kindern Orientierung und Sicherheit geben. Somit kann man den Unterricht mit einem Ritual beginnen, danach erfolgt die Präsentation der Freiarbeitsmaterialien bzw. das Erlernen des neuen Lernstoffes. Anschließend erforschen die Kinder selbständig die Sprache mit dem bereitgestellten Material und die Lehrperson begleitet sie und bietet ihnen Hilfe an, wo es notwendig ist. Am Ende notieren die Kinder in ihre Hefte, womit sie gearbeitet haben und was sie gelernt haben.

\subsection{Die freie Wahl}

„Freie Arbeit mit garantierter Zeit für alle Kinder bedeutet, dass alle Kinder frei wählen können, welchen Tätigkeiten sie nachgehen bzw. welche Aufgaben sie bearbeiten wollen, ohne dass diese Absichten mit Hinweisen auf ,eigentlich Nützlicheres“ oder ,,bisher Unerledigtes“ oder ,,besser für dich“ begrenzt werden können.“(Claussen 1995: 21)

Freie Arbeit ist eine offene Unterrichtsform, die durch eine starke organisatorische Öffnung gekennzeichnet ist, die methodische und inhaltliche Öffnung bleiben meistens jedoch nur in einer mittleren Ausprägung (Peschel 2016). Somit haben die Kinder innerhalb der freien Arbeit die Möglichkeit zur freien Wahl (vgl. Brügelmann 1997, Fisgus, Kraft 2012, Freinet 2009³, Montessori 2009, Peschel 2016):

- der Sozialform: individuell, im Paar oder in einer Gruppe

- des Arbeitsplatzes: z. B. am Tisch, auf dem Boden, in der Leseecke, im Flur

- der Reihenfolge und Dauer der geplanten Aufgaben innerhalb der Freiarbeitseinheit

- $\quad$ der Hilfsmittel (falls mehrere zum selben Thema zur Verfügung stehen)

Der Personalisierungsprozess bzw. der persönliche Ansatz dringt in alle Segmente unseres Lebens ein, nur in der Bildung verankert er sich nicht, obwohl das dringend erforderlich wäre. Unter anderem bedeutet die Personalisierung des Bildungsprozesses den Kindern zu ermöglichen ihre Interessen und Stärken zu verfolgen, sowie den Unterricht an unterschiedliche Schnelligkeiten der Lernenden anzupassen (Aronica und Robinson 2015). Vieles davon kann man mit der freien Wahl in der Freiarbeit erreichen.

3 Die Originalausgabe erschien 1977 unter dem Titel „L'Itinéraire de Célestin Freinet. La libre expression dans la pédagogie Freinet". 


\subsubsection{Freiheit und ihre Grenzen}

Das freie Lernen in einer altersgemischten Schülergemeinschaft mit unterschiedlichen Deutschkenntnissen (was oft der Fall im Deutschunterricht als nichtobligatorischem Wahlfach ist), erfordert bestimmte Regeln, die den Lernenden mehrmals vorgestellt werden. Es geht um Regeln zur Rücksichtnahme auf die Bedürfnisse der anderen Kinder (z. B. leise Bewegung durch den Raum) und Regeln zur Ordnungserhaltung der vorbereiteten Umgebung (z. B. Materialien zurück auf ihren Platz bringen). Das bedeutet, dass sich die Kinder nur in einem gewissen Maße frei verhalten können. Außerdem ist meistens von jedem Material nur ein Exemplar vorhanden und die Kinder müssen warten bis es verfügbar ist bzw. sie müssen sich einer arbeitenden Schülergruppe anschließen (Helmle, Wöbcke-Hemle 2016).

\subsection{Der Arbeitsplan}

Zu Beginn jeder Freiarbeitseinheit (eine bzw. mehrere Unterrichtsstunden) werden die Aufgaben und Materialien vorgestellt, die auch in einer Arbeitsplantabelle (Beispiel: Tabelle 1), die an einem bestimmten Platz im Zimmer ausgehängt wird, eingetragen werden. Die Tabelle enthält ebenfalls die Namen der Kinder. Es geht um ein Instrument für Lehrende und Lernende, mit dem man eine zielgerechte, lehrplanbezogene und kontinuierliche Arbeit verfolgen kann (Fisgus, Kraft 2012). Wenn ein Kind eine Arbeit beendet, trägt es das in die Tabelle zu seinem Namen ein. Der Plan ist in den meisten Fällen für alle gleich (geschlossener Arbeitsplan), dennoch kann er so gestaltet werden, dass er Alternativen zur individuellen Auswahl enthält. Auch Angebote in unterschiedlichen Schwierigkeitsstufen sind möglich.

Nachdem sich die Schüler an die Arbeit nach dem Arbeitsplan gut gewöhnt haben, wäre eine stärker mitbestimmende Variante ausführbar - die Kinder entwickelten

Tabelle 1: Arbeitsplantabelle für zwei Unterrichtseinheiten in der 4. Klasse

\begin{tabular}{|c|c|c|c|c|c|c|}
\hline $\begin{array}{l}\text { FREIARBEIT } \rightarrow \\
\text { NAME: }\end{array}$ & $\begin{array}{l}\text { Ein Buch } \\
\text { lesen }\end{array}$ & $\begin{array}{l}\text { Hören } \\
\text { (CD) }\end{array}$ & $\begin{array}{l}\text { Schnelle Frage - } \\
\text { schnelle Antwort }\end{array}$ & $\begin{array}{l}\text { Arbeit mit dem } \\
\text { Wörterbuch }\end{array}$ & $\begin{array}{l}\text { Wörter } \\
\text { stempeln }\end{array}$ & Deine Wahl: \\
\hline & & & & & & \\
\hline & & & & & & \\
\hline & & & & & & \\
\hline & & & & & & \\
\hline & & & & & & \\
\hline & & & & & & \\
\hline & & & & & & \\
\hline & & & & & & \\
\hline
\end{tabular}


gemeinsam mit der Lehrperson einen Arbeitsplan (Brügelmann 1997). Diese Arbeitsweise stimmt mit dem aktiven Lernen überein, bei dem die Lernenden im Vergleich zum geleiteten Lernen eine viel aktivere Rolle übernehmen und bei dem es vorwiegend um einen selbstregulierenden Lernansatz geht, der selbstorganisiert und selbstgeplant verläuft (de Corte 2013). Zu Beginn ist es jedoch ratsam mit einem gemeinsamen Plan für die gesamte Gruppe zu starten.

\subsection{Selbstkontrolle}

Die Fehlerkontrolle wurde von Maria Montessori begründet und bedeutet eine selbstständige Auseinandersetzung mit den eigenen Fehlern. Die von ihr erstellten Materialien enthalten immer gleichzeitig auch eine Fehlerkontrolle, mit der das Kind selbstständig erkennt, ob es sich dem Ziel nähert oder von ihm abweicht. Das macht die Fehler interessant und kräftigt das Selbstvertrauen der Lernenden (Montessori 2011).

Es gibt mehrere Möglichkeiten, die Selbstkontrolle ins Material zu integrieren. Die häufigste Variante sind die Kontrollkarten. Die können entweder ein Bestandteil der Arbeitskartei sein oder disloziert von den Übungen irgendwo im Raum stehen. Die Kinder kontrollieren selbst, ob ihre Sprachstrukturen mit denen auf den Kontrollkarten übereinstimmen. Eine der wiederkehrenden Übungen im Deutschunterricht ist das Legen der Wörter zu Gegenständen/Bildern. Am Ende kontrolliert das Kind mit der Kontrollkarte, auf der der Gegenstand abgebildet ist und unter ihm das Wort steht, ob es die Wörter richtig gelegt hat.

Bei komplexeren mündlichen Sprachübungen, mit denen sich die Kinder im Tandem oder in einer Gruppe beschäftigen, gibt es eine Variation der Selbstkontrolle, an der die Lehrperson zwar beteiligt ist, dennoch nicht in den Prozess einschreitet oder sie dabei stört. Sie kann den Lernenden bei der Sprechübung zuhören und sich die Fehler notieren. Am Ende liest sie sie vor und die Kinder korrigieren sie frontal. Der Erfahrung nach motiviert eine derartige Fehlerkontrolle die Lernenden, weil sie aktiv an der Korrektur beteiligt sind, ihr jedoch nicht direkt ausgesetzt werden.

Mit der Selbstkontrolle nimmt die Verantwortung der Kinder zu, außerdem suchen sie bei der Lehrperson nur in schwierigen Fällen Hilfe (Brügelmann 1997, Freinet 2009).

\subsection{Lernen und Bewegung}

Die Bewegung weckt und aktiviert unsere mentalen Fähigkeiten. Sie integriert und verankert neue Informationen sowie Erfahrungen in die neuronalen Netzwerke. Unser Handeln, mit dem wir das Lernen, die Einsichten und uns selbst zum Ausdruck bringen, wäre ohne die Bewegung undenkbar gewesen. Außerdem erfolgt das Lernen viel leichter, 
wenn wir den Lernenden die Möglichkeit geben, sich im Unterricht zu bewegen (Hannaford 2005). Die Kinder, die oft ihre Hände bei der Arbeit benutzen, kommen geistig und körperlich gut voran (Montessori 2011).

Die Bewegung ist ein natürlicher Begleiter der Freiarbeit. Es geht um geordnete Bewegungen durch den Raum bei der Suche nach dem Arbeitsmaterial, um Bewegung bei dem Hantieren mit dem Arbeitsmaterial (z. B. Stempeln, Legematerial) und um sinnvolle Bewegungen als Ziel vieler Sprachmaterialen im Sinne von Total Physical Response. Ein praktisches Beispiel dafür sind die Aufgabenkarten, die mit ihren Aufforderungen die Lernenden direkt zur Bewegung einladen (z. B. „Geh zur Tür und mache sie auf.“). Diese sind oft ein Bestandteil vieler Würfelspiele.

\subsection{Freiarbeitsmaterial für den DaF-Unterricht mit praktischen Beispielen}

Das Material für die freie Arbeit ist begrenzt in seiner Anzahl, zielorientiert, handlungsauffordernd und es ermöglicht eine selbstständige Arbeit ohne Hilfe der Lehrperson (Peschel 2016).

Es besteht u. A. aus Arbeitskarteien, die eine Informationskarte sowie Aufgabenund Lösungskarten enthalten, damit die Kinder die Richtigkeit selbst kontrollieren können (Freinet 2009). Sie dienen der selbständigen individuellen Einübung von mündlichen und schriftlichen Sprachhandlungskompetenzen. Dies kann auch in Partnerarbeit oder in einer Gruppe ausgeführt werden. Die Arbeitskarteien sind entweder nach Themenbereichen oder nach Schwierigkeitsgrad innerhalb eines Themas sortiert.

Außer der Arbeitskarteien eignen sich als Freiarbeitsmaterialien und -Formen z. B. auch Legematerial, Arbeitsblätter, Brettspiele, Wörter und Sätze stempeln, Arbeit am PC, Arbeit mit Hörtexten und Wörterbüchern, Bücher lesen, Lesekarten, interschulische Korrespondenz, freies Schreiben oder Gestaltung von Plakaten.

Im Weiteren werden drei über mehrere Jahre in der vierten und fünften Klasse überprüften Freiarbeitsmaterialien bzw. Freiarbeitsformen, die sich als erfolgreich erwiesen, anhand Fotos vorgestellt und kommentiert:

a) Arbeitskartei: Schnelle Frage? Schnelle Antwort.

b) Wörter/Sätze stempeln

c) Selbständige Arbeit mit Hörtexten

\section{a) Arbeitskartei: Schnelle Frage? Schnelle Antwort.}

\section{Ziel}

Mündliches oder schriftliches selbständiges Üben von Fragen stellen und Fragen beantworten auf der Wort- und Satzebene. 


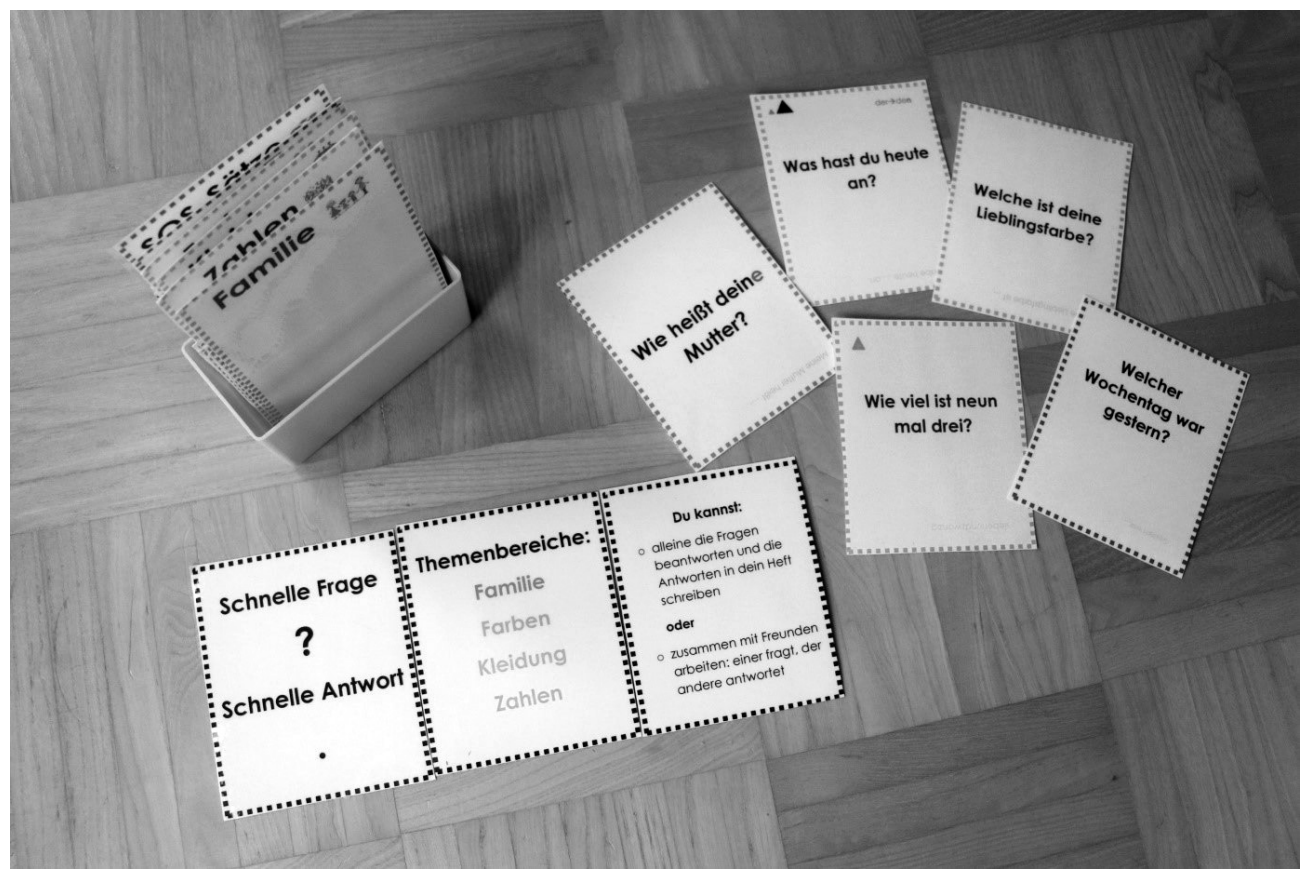

Bild 1: Arbeitskartei Schnelle Frage? Schnelle Antwort.

\section{Beschreibung}

Arbeitskartei zu unterschiedlichen vom Lehrplan vorgeschriebenen Themenbereichen (Familie, Farben, Zahlen, Kleidung) mit Antworten bzw. Antwortanfang - zur Überprüfung von Genauigkeit der verwendeten Satzstruktur und der Richtigkeit vom Gebrauch der Vokabeln. Alle Karten werden in einem Karteikasten aufbewahrt und nach Themenbereichen sortiert gehalten. Die Kartei enthält auch blanko Karten, die die Lernenden mit eigenen Fragen ergänzen können.

\section{Differenzierung und Individualisierung}

Da es möglich ist das Material sowohl schriftlich als auch mündlich, individuell oder in einer Gruppe zu verwenden, kann es auf mehreren Sprachniveaus angewandt werden. Die Fragen können weggenommen oder hinzugefügt werden, deswegen können sie individuell an das Kind angepasst werden oder das Kind entscheidet selbst, welche Fragen es beantworten möchte bzw. welche Fragen es schon beantworten kann. Wichtig ist, dass es einige Inhalte schon vorab kennengelernt hat. Fortgeschrittene können sich ihre eigenen Fragen ausdenken und sie auf blanko Karten aufschreiben. Die sind dann ein Teil der Arbeitskartei für alle Lernenden. 


\section{Vorgang}

1. Die Kinder nehmen die Arbeitskartei (üblicherweise arbeiten sie zusammen) und wählen einen Themenbereich mit dazugehörigen Fragekarten aus.

2. Eines liest eine Frage aus dem gewählten Themenbereich, die anderen Kinder beantworten sie.

3. Sie kontrollieren die Genauigkeit ihrer Antworten (Selbstkontrolle: an der unteren Seite jeder Karte (angegebene Antwort bzw. Teilantwort).

4. Nach einigen Runden tauschen die Kinder ihre Rollen.

5. *Fortgeschrittene schreiben evtl. weitere Fragen auf die blanko Karten und lassen sie von anderen Kindern beantworten.

6. Am Ende räumen sie das Material auf (sortieren die Karten nach Themenbereichen bzw. Farben) und bringen es auf seinen Platz zurück.

\section{Kommentar}

Es geht um ein einfaches, aber unter Kindern sehr beliebtes fremdsprachliches Material. Es entstand aus der Idee, dass die Lernenden im Schulalter sehr gerne zusammen arbeiten und gerne überprüfen, wie viel sie schon gelernt haben. Außerdem ermöglicht das Material den Kindern eine völlig selbständige Arbeitsweise. Der Beobachtung nach hatten sie bei dem Abfragen viel Spaß und machten Fortschritte - selbständig und mit Freude. Das Material wurde nach Bedarf immer wieder erweitert. Einige Lernende liehen sich die Arbeitskartei aus und übten damit nach dem Unterricht selbständig weiter. Es ist auch als ein Instrument bei der mündlichen Bewertung und Benotung einsetzbar.

\section{b) Wörter und Sätze stempeln}

Freinet (2009: 45) zu einem seiner Arbeitsateliers - der Schuldruckerei:

„Das stellt eine neue, sauber aufgetragene, ernsthafte, manuelle Arbeit mit einem genau bestimmten Ziel dar: das Drucken des vorher gedachten, gesprochenen, geschriebenen, gelesenen und selbsterstellten Textes."

\section{Ziel}

Wörter und Sätze richtig schreiben, Groß- und Kleinschreibung üben.

\section{Beschreibung}

Das Material besteht aus: einem Stempelset mit Buchstaben des deutschen Alphabets, zwei Stempelkissen in unterschiedlichen Farben, mehreren auf Kartonstreifen aufgeschriebenen Wörtern oder Sätzen mit markierten kritischen Stellen (rot). Eventuell können auf dem Tablett auch Bilder oder Gegenstände zu den Wörtern bereit stehen. Alle Elemente sind am praktischsten auf einem Tablett ausgelegt, damit sie von den Kindern problemlos transportiert werden können. 


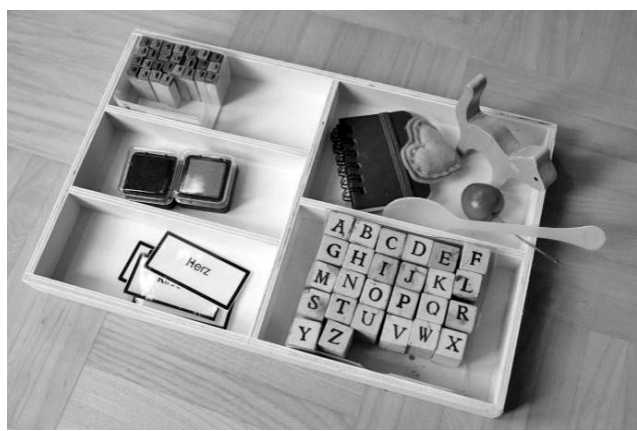

Bild 2: Tablett mit Stempelutensilien

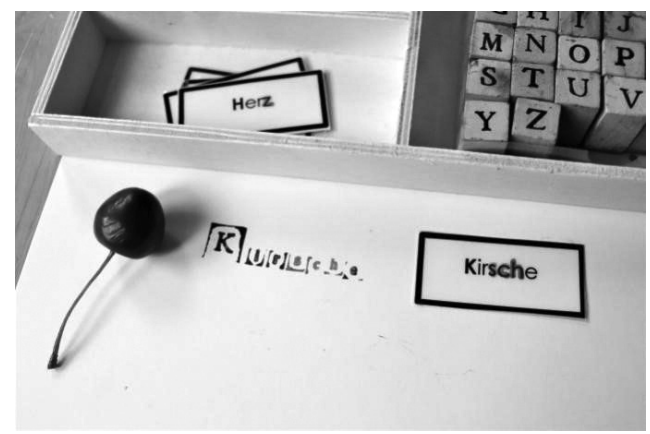

Bild 3: Gestempeltes Wort mit Selbstkontrolle

\section{Differenzierung und Individualisierung}

Das Kind oder die Lehrperson entscheiden über die Quantität der Wörter oder Sätze, die vom Kind ins Heft gestempelt werden sollen. Wegen der betonten kritischen Stellen und der Arbeit mit den Händen ist es sehr geeignet für Kinder mit einer Rechtschreibschwäche.

\section{Vorgang}

1. Das Kind holt sich das Tablett zum Wörter/Sätze Stempeln und bereitet sein Heft vor.

2. Es wählt einen Kartonstreifen und liest das Wort/den Satz mit markierten kritischen Stellen.

3. Den Kartonstreifen dreht es um und stempelt das Wort/den Satz in sein Heft. Für die kritischen Stellen kann es das Stempelkissen in rot verwenden.

4. Am Ende dreht das Kind den Kartonstreifen wieder um und vergleicht das gestempelte Wort/den gestempelten Satz mit dem Wort- bzw. Satzstreifen.

5. Zum Schluss räumt es das Material auf: sortiert die Stempel nach dem ABC, legt alle Stempel-Utensilien aufs Tablett und bringt es auf seinen Platz zurück.

\section{Kommentar}

Bei diesem Material geht es natürlich nicht um eine Schuldruckerei im Sinne Freinets, die ein viel komplexerer Unterrichtsansatz ist, sondern um ein an seiner Schuldruckerei inspiriertes Material, bei dem einige gleiche Prinzipien gelten.

Die Arbeit mit der Hand (das Abdrucken der einzelnen Buchstaben) ermöglicht den Kindern eine präzise Auseinandersetzung mit einzelnen Wörtern und deren kritischen Stellen, die schon auf dem Kartonstreifen mit rot hervorgehoben sind. Außerdem wurde im Unterricht immer wieder beobachtet, dass es zu einer hohen Konzentration bei der Arbeit mit diesem Freiarbeitsmaterial kam oder wie es Montessori nennt - zu der Polarisation der Aufmerksamkeit (Fisgus, Kraft 2012).

Die Kinder arbeiten damit meistens alleine oder mit noch einem Kind zusammen. Eine mögliche Erweiterung dieses Materials wäre das Bebildern des Wortes durch die 
Lernenden oder den entsprechenden Gegenstand zum Wort legen (Bild 3). Somit würde auch das Verstehen des abgedruckten Wortes bewiesen. Die Selbstkotrolle wäre dann eine Wort-Bild-Karte.

\section{c) Selbstständige Arbeit mit Hörtexten}

\section{Ziel}

Hörverstehen üben, Wortschatz erweitern.

\section{Beschreibung}

Für diese Aufgabe werden ein Radio oder ein Computer bzw. Tablet, ein oder mehrere geeignete Hörtexte (zur Auswahl nach dem Interesse des Lernenden) und Kopfhörer benötigt, sodass während des Hörens der Rest der frei arbeitenden Kinder nicht gestört wird. Die Arbeit verläuft individuell oder im Paar.

\section{Differenzierung und Individualisierung}

Das Kind kann sich den Hörtext nach Bedarf mehrmals anhören und die Audioaufnahme anhalten, wenn es möchte. Aus dem gehörten Text können die Lernenden z. B. fünf Wörter, die sie erkennen, ins Heft unter den Titel „Diese Wörter kenne ich schon“ schreiben und ihre Bedeutung im (Online-)Wörterbuch überprüfen. Falls mehrere Hörtexte zur Verfügung stehen, kann das Kind einen nach seinem Interesse alleine oder mit der Lehrpersonhilfe aussuchen.

\section{Vorgang}

1. Das Kind setzt sich in die Hörecke, wo ein PC/Tablet/Radio mit Kopfhörern ist. Es sucht sich einen Hörtext (als CD bzw. Datei) aus.

2. Es hört sich den Hörtext mit Kopfhörern beliebig oft an und hält die Aufnahme an, wo es nötig ist.

3. Eventuell können in der Hörecke auch Übungen zum Hörverstehen bereitstehen, die das Kind während oder nach dem Hören macht.

4. Nach der vollendeten Arbeit bereitet das Kind die Hörecke für das Nächste vor.

\section{Kommentar}

Eine wertvolle Übung für Fremdsprachelernende, denn sie ermöglicht eine Lernsituation im eigenen Lernrhythmus, die im Frontalunterricht wegen der Schülerzahl oft nicht umsetzbar ist. Der Beobachtung nach gingen die Kinder sehr unterschiedlich mit dieser Übung um. Einige hörten sich den Text nur einmal an, andere mehrmals und vertieften sich in die Arbeit, indem sie bekannte Wörter ausschrieben und deren Bedeutung nachprüften. Um das Hörverstehen durch Schüler selbständig kontrollieren zu lassen, könnten einige kurze Übungen zum Umkreisen, Ankreuzen, einzelne Wörter einsetzen oder zum 
Bilder bemalen angeboten werden. Die Lösung sollte irgendwo im Raum ausgehängt sein.

3

\section{FREIARBEIT UND DER NATIONALE LEHRPLAN FÜR DIE ZWEITE FREMDSPRACHE ALS NICHTOBLIGATORISCHES WAHLFACH IN DER 4. UND 5. KLASSE}

Der slowenische nationale Lehrplan für zweite Fremdsprache als nichtobligatorisches Wahlfach von der 4. bis zur 9. Klasse stimmt an vielen Stellen mit den Prinzipen der Freiarbeit überein. Die Lehrperson dieses Wahlfachs steht oft vor der Herausforderung eine äußerst heterogene Lerngruppe unterrichten zu müssen, was nur durch geeignete Lehransätze und innere Differenzierung abgemildert werden kann. Die Autorinnen des Lehrplans empfehlen daher ganzheitliche schülerzentrierte Lehransätze und -Methoden, die es jedem Kind ermöglichen im eigenen Tempo zu lernen und eigene Lernstrategien zu entwickeln (Pevec Semec et al. 2013).

Die Lehrende dieses Wahlfaches sind Mentoren der Lernenden, die ebenfalls an der Unterrichtsplanung beteiligt sein sollen, was ein wichtiges Prinzip des offenen Unterrichts ist (vgl. Brügelmann 1997). Die Tatsache, dass sich die Kinder freiwillig für die Teilnahme am DaF-Unterricht entscheiden, ist eine gute Voraussetzung für die Freiarbeit, bei der sowohl das Kind, als auch die Lehrperson die Verantwortung für einen gelungen Lehr-Lern-Prozess tragen.

Im Lehrplan wird an vielen Stellen erwähnt, die Lernenden zu Selbstreflexion, Selbstevaluation, Selbstkorrektur und Selbstbewertung innerhalb des Lernprozesses aufzufordern, was insbesondere mit der Selbstkontrolle in der Freiarbeit übereinstimmt.

Die Autorinnen des Lehrplans warnen, dass es bei den Lernenden zu Demotivation für das Lernen oder sogar zu störendem Verhalten seitens der Kinder kommen könnte, wenn es für ihr Sprachniveau kein geeignetes Aktivitätsangebot gibt (Pevec Semec et al.). Außerdem kann die ,Gleichheit“ für alle Lernenden zu Über- bzw. Unterforderung führen (Peschel 2016). Binnen der freien Wahl in der Freiarbeit können sich die Kinder ihren Bedürfnissen entsprechendes Sprachmaterial, die Sozialform sowie das Arbeitstempo aussuchen und den Arbeitsplatz frei bestimmen, was das Lernen auf unterschiedlichen Niveaus wesentlich leichter machen kann.

4

\section{SCHLUSSBEMERKUNGEN (MÖGLICHKEITEN UND GRENZEN DER FREIARBEIT IM DAF-UNTERRICHT)}

Freiarbeit im DaF-Unterricht ersetzt nicht die anderen Lehrformen, wie etwa den Frontalunterricht, der z. B. für die Behandlung von literarischen Texten oder Liedersingen 
geeigneter ist. Es geht um eine Handlungsform, die zu Beginn besonders gut in Übungsphasen anwendbar ist. Wichtig ist, dass sie schrittweise eingeführt wird, denn die Lernenden müssen sich erst an diese Arbeitsform gewöhnen, deswegen ist der Einstieg über ein bekanntes didaktisches Material sinnvoll (Fisgus, Kraft 2012). Somit können die ersten Freiarbeitsstunden mit Arbeitsblättern oder Übungen aus dem Arbeitsbuch, mit Brettspielen, die die Kinder schon kennen, und mit einem neuen Material (z. B. der Arbeitskartei zu einem bestimmten Thema) begonnen werden.

Die Stundentafel schreibt vor, dass Deutsch als nichtobligatorisches Wahlfach in der 4. und 5. Klasse zwei Unterrichtseinheiten pro Woche unterrichtet wird. In den meisten Fällen ist es wegen der Zeitorganisation so, dass die Kinder den Deutschunterricht an zwei unterschiedlichen Wochentagen, üblicherweise nach ihrem Pflichtunterricht, erhalten. Einerseits können die Kinder schon müde und weniger lernbereit sein, andererseits ermöglicht ihnen die Freiarbeit als offene Unterrichtsform mehr Freiraum und Ausdrucksmöglichkeit, was eine motivierende Wirkung haben kann. Wenn der Deutschunterricht an einem Wochentag zwei aufeinanderfolgende Unterrichtseinheiten dauert, gibt das der Lehrperson und den Lernenden die Möglichkeit sich genauer und kontinuierlicher mit der Freiarbeit auseinanderzusetzen. Im Gegenfall muss die Freiarbeit gewöhnlich auf mehrere Unterrichtseinheiten verteilt werden. Allerdings gibt es laut dem slowenischen Grundschulgesetz noch die Option der flexiblen Stundentafel, die es ermöglicht die Unterrichtsstunden in der Woche anders als vorgeschrieben zu verteilen (ZOsn 2016, 29. Artikel).

Die Freiarbeit basiert auf dem Sprachmaterial, das entweder von der Lehrperson erstellt oder seitens der Schule angeschafft werden muss. Das eine ist mit dem Zeitaufwand, das andere mit den finanziellen Möglichkeiten der Schule verbunden. Überdies kann die Freiarbeitsumgebung im Gastklassenzimmer erst vor Unterrichtsbeginn vorbereitet werden. Eine Möglichkeit wäre eine Deutschecke/einen Deutschschrank oder ein Deutschregal im Gastklassenzimmer aufzustellen, wo die Materialien aufbewahrt würden. Jedoch muss der Lehrende sie meistens von Klasse zu Klasse tragen.

Es geht daher um eine persönliche Entscheidung, ob die Lehrperson dazu bereit ist Neues zu versuchen, für die vorbereitete Umgebung zu sorgen, sich im Freiarbeitsprozess zurückzunehmen, sowie den Kindern mehr Mitbestimmung zu ermöglichen (Fisgus, Kraft 2012).

\section{BIBLIOGRAPHIE}

ARONICA, Lou/Ken ROBINSON (2015) Kreativne šole. Nova Gorica: Eno. BRÜGELMANN, Hans (1997) Die Öffnung des Unterrichts muss radikaler gedacht, aber auch klarer strukturiert werden. 9. Mai 2019. https://userpages.uni-koblenz. de/ proedler/bruegelmann.htm. 
CLAUSSEN, Claus (1995) Freie Arbeit als Element eines Konzepts der Öffnung von Schule und Unterricht. C. Claussen (Hrsg.), Handbuch Freie Arbeit. Konzepte und Erfahrungen. Weinheim und Basel: Beltz Verlag, 13-23.

DE CORTE, Eric (2013) Zgodovinski razvoj razumevanja učenja. H. Dumont et al. (Hrsg.), O naravi učenja. Uporaba raziskav za navdih prakse. Ljubljana: ZRSŠ, MIZŠ, OECD, 37-59.

FISGUS, Christel/Gertud KRAFT (2012) Hift mir, es selbst zu tun! Montessoripädagogik in der Regelschule. Donauwörth: Auer.

FREINET, Elise (2009) Erziehung ohne Zwang. Der Weg Célestin Freinets. Stuttgart: Klett-Cotta.

HANNAFORD, Carla (2005) Smart Moves: Why Learning is not all in your head. Salt Lake City: Great River Books.

HARRER, Margareta (2015) Konstruktivismus als Wirkmechanismus der MontessoriPädagogik - Montessori-Pädagoginnen im Praxisfeld Öffentliche Volksschule. Das Kind 57, 85-99.

HELMLE, Thomas/Petra WÖBCKE-HELMLE (2016) Praxisbuch Kosmische Erziehung. Mit Interesse lernen. Freiburg in Bresigau: Herder Verlag.

GÖNDÖR, Jürgen (2013) Offener Unterricht: ...hier lerne ich was ich will. Von der Freiheit, das eigene Lernen im Unterricht selbst zu gestalten. Borsdorf: Edition Winterwork.

MARENTIČ POŽARNIK, Barica (2018) Psihologija učenja in pouka. Od poučevanja $k$ učenju. 2. prenovljena izd. Ljubljana: DZS.

MONTESSORI, Maria (2011) Srkajoči um. Ljubljana: Uršulinski zavod za vzgojo, izobraževanje in kulturo.

MONTESSORI, Maria (2009) Skrivnost otroštva. Ljubljana: Uršulinski zavod za vzgojo, izobraževanje in kulturo.

PESCHEL, Falko (2016) Offener Unterricht. Idee, Realität, Perspektive und ein praxiserprobtes Konzept zur Diskussion. Teil 1. Allgemeindidaktische Überlegungen. J. Bennack (Hrsg.), Basiswissen Grundschule. Baltmannsweiler: Schneider Verlag Hohengehren.

PEVEC SEMEC, Katica/ Liljana KAČ/Neva ŠEČEROV/Simona CAJHEN (Hrsg.) (2013) Učni načrt. Program osnovna šola. Drugi tuji jezik v 4. do 9. razredu: neobvezni izbirni predmet. 1. Aufl. Ljubljana: Ministrstvo za izobraževanje, znanost in šport: Zavod Republike Slovenije za šolstvo.

Zakon o osnovni šoli. https://zakonodaja.sio.si/predpis/zakon-o-osnovni-soli-zosn/ (14. Mai 2019). 


\section{POVZETEK}

\section{Prosto delo pri pouku nemščine kot tujega jezika na razredni stopnji v osnovni šoli - možno- sti in omejitve}

Pouk nemščine kot neobveznega izbirnega predmeta $\mathrm{v}$ slovenskih osnovnih šolah na razredni stopnji pogosto poteka $\mathrm{v}$ starostno mešanih skupinah in $\mathrm{v}$ skupinah učencev $\mathrm{z}$ različnim predznanjem tujega jezika. To predstavlja izziv tako za učitelja kot tudi za učence. Tudi učni načrt za ta izbirni predmet pogosto omenja tovrstno problematiko. Avtorice učnega načrta zato predlagajo celostno učenje in poučevanje s pristopi, ki učence vključujejo v učni proces od načrtovanja do evalvacije. Prosto delo je didaktični pristop, ki s svojimi načeli proste izbire, samostojne kontrole, gibanja med poukom in z različnimi jezikovnimi materiali v mnogih vidikih olajša proces učenja in poučevanja v omenjenih učnih skupinah, saj pri delu omogoča visoko stopnjo notranje diferenciacije. Gre za obliko odprtega pouka (nem. Offener Unterricht), ki izvira iz reformske pedagogike, pri katerem je v ospredju samostojno, pretežno individualno učenje v pripravljenem okolju. Namen tega prispevka je predstaviti prosto delo pri pouku nemščine kot neobveznega izbirnega predmeta iz teoretičnega in praktičnega vidika, upoštevajoč možnosti in omejitve uporabe tega pristopa $\mathrm{V}$ javni osnovni šoli. V prispevku so poleg tega natančno predstavljeni preizkušeni materiali za prosto delo pri pouku tujega jezika na razredni stopnji, ki jih učitelji tujih jezikov lahko izdelajo in uporabijo pri svojem pouku.

Ključne besede: pouk nemščine kot tujega jezika, prosto delo, kontrola napake, jezikovni material, Montessori, Freinet

\section{ABSTRACT \\ Free work in German as a foreign language classes at elementary school - possibilities and limitations}

German as a foreign language class is an optional subject at elementary schools in Slovenia, one that is often attended by students of mixed ages and with varying knowledge of the language, and this is challenging for both teachers and students. The national curriculum for this subject often refers to this issue as well. For this reason the authors of the curriculum suggest a holistic approach to teaching and learning, one that involves the students in the learning process, from planning to evaluation. Free work is a didactic approach which makes the teaching and learning process easier due to its principles of free choice, self-correction, movement in learning and language materials, which together enable high levels of inner differentiation. It is a form of Open Learning (Ger. Offener Unterricht) that follows the line of New Education which puts in focus self-regulated, mainly individual learning in a prepared environment. The aim of this article is to present free work in 
German language classes from a theoretical and practical point of view, considering the limitations and possibilities of this approach when applied in public schools. Furthermore, the article presents proven free work materials for young foreign language learners that can be produced and used by foreign language teachers.

Keywords: German as foreign language class, free work, control of error, language material, Montessori, Freinet

\section{ZUSAMMENFASSUNG}

Deutsch als nichtobligatorisches Wahlfach wird in den slowenischen Grundschulen oft in altersgemischten Schülergruppen mit unterschiedlichen Deutschkenntnissen unterrichtet. Das bedeutet sowohl eine Herausforderung für die Lehrperson als auch für die Lernenden. Auf diese Problematik wird auch im nationalen Lehrplan für das Wahlfach an vielen Stellen eingegangen. Aus dem Grund schlagen die Autorinnen des Lehrplans ganzheitliche Lehransätze vor, die die Lernenden in den Unterricht miteinbeziehen, und zwar von der Unterrichtsplannung bis zur dessen Evaluation. Freiarbeit ist ein didaktischer Lehransatz, der mit seinen Prinzipien der freien Wahl, Selbstkontrolle, Bewegung im Unterricht und mit seinen Freiarbeitsmaterialien den Lehr-LernProzess in einigen Hinsichten erleichtern kann, da er in erwähnten Schülergruppen ein hohes Maß an innerer Differenzierung der Arbeit ermöglicht. Es geht um eine Form des offenen Unterrichts mit den Wurzeln in der Reformpädagogik, bei dem selbständiges, vorwiegend individuelles Lernen in einer vorbereiteten Umgebung im Vordergrund steht. Das Anliegen dieses Beitrags ist es die Freiarbeit im DaF-Unterricht theoretisch und praxisnah, mit den Möglichkeiten und Grenzen der Umsetzung in einer Regelschule, vorzustellen. Im Beitrag werden konkrete und erprobte Beispiele der Freiarbeitsmaterialien für den Fremdsprachenunterricht in der Grundschule detailliert vorgestellt und kommentiert. Sie können von den Fremdsprachenlehrenden erstellt und in ihrem Unterricht verwendet werden.

Schlüsselwörter: DaF-Unterricht, Freiarbeit, Fehlerkontrolle, Sprachmaterial, Montessori, Freinet 